\title{
Active Longitudes and Flip-Flops in Binary Stars
}

\author{
Heidi Korhonen ${ }^{1}$ and Silva P. Järvinen ${ }^{1,2}$ \\ ${ }^{1}$ Astrophysikalisches Institut Potsdam, An der Sternwarte 16, D-14482 Potsdam, Germany \\ ${ }^{2}$ Astronomy Division, P.O.Box 3000, FI-90014 University of Oulu, Finland
}

\begin{abstract}
We present results from an investigation where the long-term photometry of several magnetically active RS CVn binaries is studied to see whether or not they show permanent active longitudes and the flip-flop phenomenon. We confirm that it is very common for the active regions to occur on permanent active longitudes. Many of our target stars also show clear flip-flop phenomenon, but often the data set is not long enough for reliable determination of the flip-flop period.
\end{abstract}

Keywords. stars: activity, stars: late-type, stars: spots

\section{Introduction}

In many active stars the spots concentrate on two permanent active longitudes which are $180^{\circ}$ apart. In some of these stars the dominant part of the spot activity changes the longitude every few years. This so-called flip-flop phenomenon was first reported in the early 1990's in the single, late-type giant FK Com (Jetsu et al. 1993). Since then flipflops have been reported also on binary stars (e.g., Berdyugina \& Tuominen 1998), young solar type stars (e.g., Järvinen et al. 2005) and the Sun itself (Berdyugina \& Usoskin 2003). Even though this phenomenon has been detected on many different kinds of active stars, still only about ten stars are known to exhibit this effect. Therefore no statistically significant correlation between the stellar parameters and the flip-flop phenomenon can be carried out.

\section{Sample and methods}

Korhonen \& Elstner (2005) investigated the long-term light-curve behaviour caused by the flip-flop phenomenon using dynamo models. In the current investigation we have studied in detail stars showing similar patterns as the flip-flop signatures seen by Korhonen \& Elstner. The observations are mainly old, already published photometry, but we have also received new data. We have up to now investigated 14 stars, 9 of them binaries, using the light-curve inversion techniques.

In this technique the phased light curves are used for obtaining spot filling factor maps of the stellar surface with an inversion technique using the Maximum Entropy Method. The exact formulation follows closely the one by Lanza et al. (1998). Figure 1 shows an example of a light-curve for UZ Lib and a corresponding spot filling factor map.

\section{Results}

The phases of the spots can be determined from the spot filling factor maps obtained with light-curve inversions. In Figure 2 the results for XX Tri and UZ Lib are shown. 

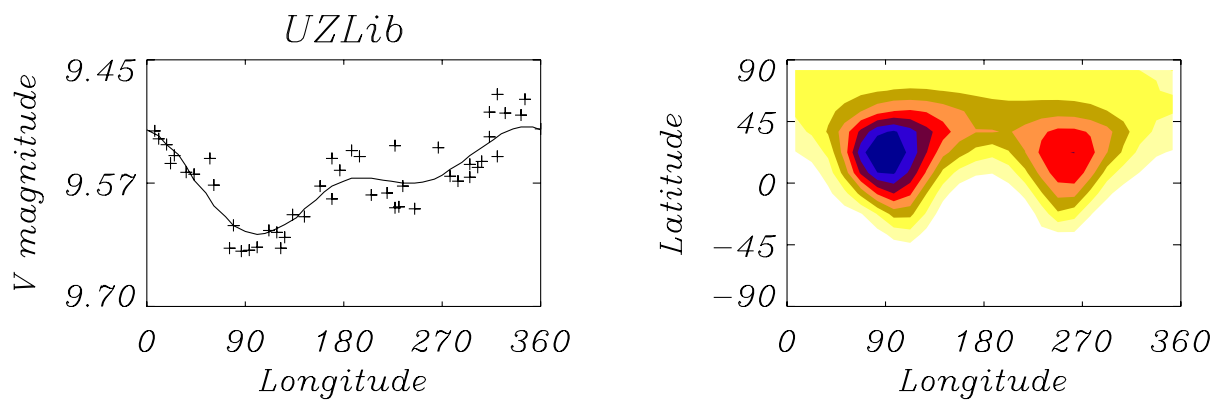

Figure 1. Results from the light-curve inversion for UZ Lib in June-July 1998. The $V$ band observations are given by crosses and the inversion result is marked with a line. In the maps the darker areas mean larger spot filling factor.
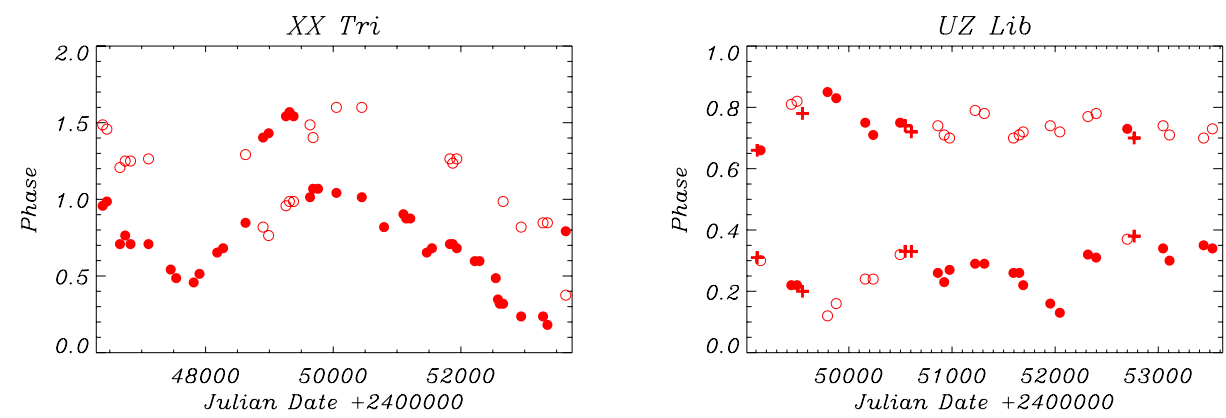

Figure 2. Phases of the spots in XX Tri and UZ Lib determined from the light-curve inversions plotted versus the Julian Date. Filled circles denote the larger spots, open circles the secondary spots and the cross a case where the two spots are equally strong.

The major spots are given as filled circles, minor ones as open circles and spots of equal size are marked with crosses.

Our investigation confirms that many active stars show permanent active longitudes, and that also the flip-flop phenomenon itself is fairly common. But unfortunately in many cases the length of the flip-flop cycle cannot be reliably determined, due to the fact that for pinpointing the length of the flip-flop cycle we would need quite a long sequence of observations and this is often not the case. Table 1 contains the binary star targets and also gives information on active longitudes and flip-flop phenomenon on these stars. A plus sign in the active longitude column means that permanent active longitudes are detected in this star. In the last column information on the flip-flop phenomenon is given: - sign means no flip-flops were detected, + sign means that flip-flops were detected, but no reliable estimate on the cycle length could be obtained. If a flip-flop cycle was detected, the full cycle length in years is given.

From the 14 stars we have investigated, we have managed to obtain an estimate of the flip-flop period for 4 binaries and 1 single star. In Figure 3 we plot the radius against the flip-flop period for these 5 stars and the previously known flip-flop stars. It seems that the long flip-flop periods ( $>10$ years) tend to occur in large stars (radius $>5 \mathrm{R}_{\odot}$ ), whereas the shorter flip-flop periods do not seem to depend on the radius. Also, it seems that the possible range of flip-flop periods for the binary stars (approximately 3.5-20 years) is much larger than for the single stars (approximately 3.5-7 years). 
Table 1. The star sample of binary stars investigated in this study. The name of the star, spectral and variability type, stellar radius and rotation period are given together with our results on active longitudes and flip-flops.

\begin{tabular}{lllllccc}
\hline \multicolumn{1}{c}{ Star } & HD & $\begin{array}{c}\text { Spectral } \\
\text { type }\end{array}$ & $\begin{array}{c}\text { Variability } \\
\text { type }\end{array}$ & $\begin{array}{c}\text { Radius } \\
{\left[\mathrm{R}_{\odot}\right]}\end{array}$ & $\begin{array}{c}P_{\text {rot }} \\
{[\text { days] }}\end{array}$ & $\begin{array}{c}\text { Active } \\
\text { longitudes }\end{array}$ & $\begin{array}{c}\text { flip-flops } \\
\text { [years] }\end{array}$ \\
\hline XX Tri & HD 12545 & K0 III & RS CVn & 8.0 & 23.98 & + & + \\
UX Ari & HD 21242 & K0 IV & RS CVn & 5.78 & 6.44 & + & + \\
IL Hya & HD 81410 & K1 III & RS CVn & 8.1 & 12.73 & + & 10.1 \\
HU Vir & HD 106225 & K1 IV & RS CVn & 5.7 & 10.39 & + & 11.4 \\
IN Com & HD 112313 & G5 III-IV & RS CVn & 7.8 & 5.92 & $?$ & - \\
HK Lac & HD 209813 & K0 III & RS CVn & 9.5 & 24.43 & + & - \\
V833 Tau & HD 283750 & K5 V & BY Dra & 0.77 & 1.79 & - & - \\
V1355 Ori & HD 291095 & K2 VI-V & RS CVn & 4.1 & 3.86 & + & 3.4 \\
UZ Lib & & K0 III & RS CVn & 6.3 & 4.77 & + & 16 \\
\hline
\end{tabular}

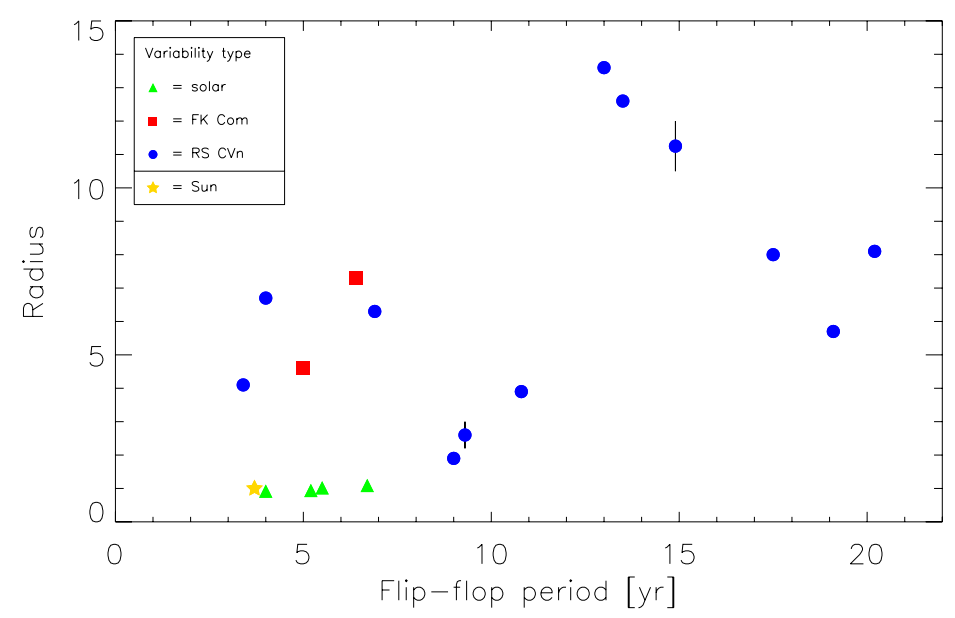

Figure 3. Stellar radius plotted against the flip-flop period for the newly discovered and previously known flip-flop stars

\section{Acknowledgements}

We would like to thank K.G. Strassmeier for giving us some of his unpublished data to be used in this study. This project has been supported by German Deutsche Forschungsgemeinschaft, DFG grant KO 2320/1-2. SPJ acknowledges support from the Väisälä Foundation, Finland.

\section{References}

Berdyugina, S.V. \& Tuominen, I. 1998, A\& $A$ 336, 25

Berdyugina, S.V. \& Usoskin, I.G. 2003, A\&A 405, 1121

Järvinen, S.P., Berdyugina, S.V., Tuominen, I., Cutispoto, G., \& Bos, M. 2005, A\&A 432, 657

Jetsu, L., Pelt, J., \& Tuominen, I. 1993, A\&A 278, 449

Korhonen, H. \& Elstner, D. 2005, A\&A 440, 1161

Lanza, A.F., Catalano, S., Cutispoto, G., Pagano, I., \& Rodonò, M. 1998, A\&A, 332, 541 